\title{
Article \\ Depolarization of Vector Light Beams on Propagation in Free Space
}

\author{
Nikolai Petrov
}

check for

Citation: Petrov, N. Depolarization of Vector Light Beams on Propagation in Free Space. Photonics 2022, 9, 162. https://doi.org/10.3390/ photonics 9030162

Received: 7 February 2022

Accepted: 4 March 2022

Published: 6 March 2022

Publisher's Note: MDPI stays neutral with regard to jurisdictional claims in published maps and institutional affiliations.

Copyright: (C) 2022 by the author. Licensee MDPI, Basel, Switzerland. This article is an open access article distributed under the terms and conditions of the Creative Commons Attribution (CC BY) license (https:// creativecommons.org/licenses/by/ $4.0 /)$.
Scientific and Technological Centre of Unique Instrumentation of the Russian Academy of Sciences, 15 Butlerova Str., 117342 Moscow, Russia; petrovni@mail.ru

\begin{abstract}
Nonparaxial propagation of the vector vortex light beams in free space was investigated theoretically. Propagation-induced polarization changes in vector light beams with different spatial intensity distributions were analyzed. It is shown that the hybrid vector Bessel modes with polarization-OAM (orbital angular momentum) entanglement are the exact solutions of the vector Helmholtz equation. Decomposition of arbitrary vector beams in the initial plane $\mathrm{z}=0$ into these polarization-invariant beams with phase and polarization singularities was used to analyze the evolution of the polarization of light within the framework of the $2 \times 2$ coherency matrix formalism. It is shown that the 2D degree of polarization decreases with distance if the incident vector beam is not the modal solution. The close relationship of the degree of polarization with the quantum-mechanical purity parameter is emphasized.
\end{abstract}

Keywords: polarization; degree of polarization; depolarization; purity parameter; vortex light; vector Bessel modes; phase and polarization singularities; polarization invariance; entropy

\section{Introduction}

The polarization of light reflects the vector nature of electromagnetic fields and plays a very important role in optics and physics of the light-matter interaction [1-3]. The polarization properties of fields should be taken into account in many problems of light propagation in various media. It is known that the polarization of a plane wave in a homogeneous and isotropic non-dispersion medium does not change during propagation. Although a polarized plane wave propagates in free space without changing its polarization state, structured light beams undergo depolarization and a change in the polarization state during propagation due to diffraction and spin-orbit interaction. Significant changes in the state of polarization and the degree of polarization occur during the propagation of radiation in inhomogeneous media. Physically, depolarization occurs due to a decrease in the degree of correlation between the different field components. In [4,5] depolarization of light in randomly inhomogeneous media was investigated. Two mechanisms of depolarization were analyzed: diffractional and geometrical, which are caused by diffraction and the Rytov rotation [6,7] of the polarization vector, respectively. In single-mode optical fibers, the change in the input polarization is usually due to nonlinearity and birefringence of the medium [8-10]. However, depolarization takes place also in optical waveguides without birefringence. In particular, it was shown in [11] that the polarization degree of the linearly polarized light in a graded-index isotropic optical fiber decreases with increasing distance. In [12], it was shown theoretically that the polarization degree of the linearly polarized light in an isotropic optical fiber with parabolic distribution of refractive index decreases with increasing distance due to the Rytov rotation of the polarization vector, but the degree of polarization of circularly polarized light is retained with increasing distance. However, in the experiments [13], preservation of the polarization degree for circularly polarized light in an isotropic optical fiber has been not observed. In [14-16], the quantum-mechanical formalism of coherent states was applied to study the evolution of polarization in a multimode 
isotropic graded-index medium. It was shown that the effects of diffraction and spin-orbit interaction are responsible for depolarization of light in an isotropic graded-index medium. The rotation of the plane of linear polarization during propagation in an optical fiber is considered. It is shown that the axial displacement and the angle of tilt of the incident beam to the fiber axis influence on the rotation angle of the polarization plane. Intrafibre rotation of the plane of polarization was also demonstrated in [17].

In past decades, there has been considerable interest in studying changes in the degree of polarization of electromagnetic beams on propagation in free space [18-26]. It has been shown that, in general, the degree of polarization varies with propagation even in free space [18,19]. In [25], the possibility of controlling the degree of polarization of light during propagation in free space by changing the coherence of the light source was demonstrated experimentally. The results were in good agreement with the theory [24]. In some cases, the spectral degree of polarization remains propagation-invariant along the axis of a Gaussian Shell-model beam $[27,28]$. Recently, the study of the propagation and focusing of nonuniformly totally polarized beams has been of great interest [29-34]. Of particular interest is the study of the invariance on propagation of the characteristics of vector beams, such as the state and degree of polarization [35-38]. In [27,36,37], the conditions under which the polarization invariance is observed in the propagation of partially coherent electromagnetic beams are analyzed. It is known that the spirally polarized beams $[29,30,39,40]$ remain invariant in polarization during propagation. Note that the radial and azimuthal polarizations are the particular cases of the spirally polarized beams.

In recent years, the study of the vector vortex beams with the phase and polarization singularities has been of particular interest [41-46]. Various types of cylindrical vector beams (CVBs) with helical wavefronts and spatially nonuniform state of polarization, such as Laguerre-Gauss (LG) beams which combine spin and orbital angular momentum (OAM), were proposed. It should be noted that cylindrically polarized LG beams can be considered as modal solutions in free space only in the paraxial approximation. Paraxial and nonparaxial propagation properties of cylindrically polarized beams have been considered in [47,48]. In [49-51], generation of arbitrary vector vortex beams on hybrid-order Poincaré sphere was investigated. In [52], an overview of the latest results on the generation and observation of polarization singularities in metaphotonics is presented. A review on polarization optics and polarimetry for recent biomedical and clinical applications is presented in [53]. In [54], the phase and polarization singularity sheets using metasurfaces experimentally are realized. Recent developments of wave field multidimensional manipulations based on artificial microstructures are presented in [55]. In [56] the spin-decoupled metasurface for simultaneous detection of SAM (spin angular momentum) and OAM via momentum transformation was proposed.

Various approaches to generate vector vortex beams have been proposed [31], such as interferometry, subwavelength gratings, conical Brewster prism, twisted nematic liquid crystals, etc. In [51], to generate an arbitrary vector vortex beam on the hybrid-order Poincaré sphere, a simple approach using the combination of an inhomogeneous birefringent $q$-plate and a spiral phase plate is proposed. A passive device consisting of a pair of polarization-selective cylindrical lenses for generating cylindrical vector beams with arbitrary azimuthal and radial topological charges is demonstrated in [44].

The usual description of polarization is based on the Stokes parameters or on $2 \times 2$ polarization matrices. In $[57,58]$ the unified theory of coherence and polarization is formulated which can be used to study the changes of polarization at propagation of random electromagnetic beams. Usually, the calculations of the coherence matrix elements are carried out in a paraxial approximation. However, for tightly focused beams, nonparaxial effects become significant.

In this paper, a theoretical analysis of the propagation of polarized vortex light beams in free space is carried out using the vector mode decomposition method. It is found that the hybrid vector Bessel modes with radial and azimuthal indices are the solutions of the Maxwell equations. Polarization-invariant vector Bessel beams with phase and polarization 
singularities which combine spin and OAM are proposed. Change in the polarization state and the degree of polarization on propagation is shown for an arbitrary incident beam that is not a modal solution of the Maxwell equations.

\section{Problem Formulation}

The equation describing the propagation of monochromatic light in free space for a vector electric field $\vec{E}$ can be obtained from Maxwell's equations as

$$
\nabla \times \nabla \times \vec{E}-k^{2} \vec{E}=0,
$$

where $\vec{E}(r, \varphi, z)=\left(\vec{e}_{\perp}+e_{z} \vec{z}\right) \exp (i \beta z), \vec{e}_{\perp}=e_{r} \vec{e}_{r}+e_{\varphi} \vec{e}_{\varphi}, e_{z}=\frac{i}{\beta} \vec{\nabla}_{\perp} \vec{e}_{\perp}, k=2 \pi / \lambda$ is the wavenumber, and $\beta$ is the propagation constant.

The radial, azimuthal and longitudinal components of the electric field, defined in cylindrical polar coordinates $r, \varphi, z$, are described by the equations:

$$
\begin{gathered}
\frac{1}{r} \frac{\partial}{\partial r}\left(r \frac{\partial e_{r}}{\partial r}\right)-\frac{1}{r^{2}} e_{r}-\frac{2}{r^{2}} \frac{\partial e_{\varphi}}{\partial \varphi}+\frac{1}{r^{2}} \frac{\partial^{2} e_{r}}{\partial \varphi^{2}}+\frac{\partial^{2} e_{r}}{\partial z^{2}}+k^{2} e_{r}=0, \\
\frac{1}{r} \frac{\partial}{\partial r}\left(r \frac{\partial e_{\varphi}}{\partial r}\right)-\frac{1}{r^{2}} e_{\varphi}+\frac{2}{r^{2}} \frac{\partial e_{r}}{\partial \varphi}+\frac{1}{r^{2}} \frac{\partial^{2} e_{\varphi}}{\partial \varphi^{2}}+\frac{\partial^{2} e_{\varphi}}{\partial z^{2}}+k^{2} e_{\varphi}=0, \\
\frac{1}{r} \frac{\partial}{\partial r}\left(r \frac{\partial e_{z}}{\partial r}\right)+\frac{1}{r^{2}} \frac{\partial^{2} e_{z}}{\partial \varphi^{2}}+\frac{\partial^{2} e_{z}}{\partial z^{2}}+k^{2} e_{z}=0 .
\end{gathered}
$$

Below we consider the beams with spatial transverse dimensions significantly exceeding the wavelength, so the longitudinal field component $e_{z} \ll e_{\perp}$ can be neglected because the electromagnetic field in this case is transverse. For such fields, the polarization is well described by Stokes parameters determined from the $2 \times 2$ coherency matrix [1].

Cylindrical hybrid vector Bessel beams with phase (scalar) and polarization (vector) singularities are the exact solutions of the vector two-dimensional Helmholtz Equation (2):

$$
\vec{e}(r, \varphi, z)=\left(\begin{array}{c}
e_{r} \\
e_{\varphi}
\end{array}\right)=\frac{1}{2} \psi_{p l}(r)\left\{e^{i l \varphi+i \varphi_{0}}\left(\begin{array}{c}
1 \\
-i
\end{array}\right) e^{-i \varphi}+e^{-i l \varphi+i \varphi_{0}}\left(\begin{array}{c}
1 \\
i
\end{array}\right) e^{i \varphi}\right\} e^{i \beta_{p l} z},
$$

where $\psi_{p l}(r)=A J_{l}\left(\mu_{p l} r / R_{0}\right), A=1 /\left(\sqrt{\pi} R_{0} J_{l+1}\left(\mu_{p l}\right)\right), \beta_{p l}=k \sqrt{1-\left(\frac{\mu_{p l}}{k R_{0}}\right)^{2}}, \mu_{p l}$ are the positive zeros of the Bessel function $J_{l}(z) ; p=1,2, \ldots ; l=0,1,2, \ldots ; \varphi_{0}$ is the initial angle.

Solutions (3) are the combination of Bessel functions of different order, and they consist of different polarization states. Vector modes are hybrid, i.e., they include both components of the field. Note that the solution (3) satisfies the Maxwell's divergence equation $\nabla \cdot \vec{e}(r, \varphi, z)=0$.

It follows from the orthogonality condition for Bessel functions [59]

$$
\int_{0}^{R_{0}} J_{m}\left(\mu_{i} r / R_{0}\right) J_{m}\left(\mu_{j} r / R_{0}\right) r d r=\frac{R_{0}^{2}}{2}\left[J_{m+1}\left(\mu_{i}\right)\right]^{2} \delta_{i j},
$$

that the normalized functions $\psi_{p l}(r)$ satisfy the equation

$$
\int_{0}^{2 \pi} \int_{0}^{R_{0}} \psi_{p l}^{*}(r, \varphi) \psi_{p l}(r, \varphi) r d r d \varphi=1 .
$$

These solutions form a complete set of mutually orthogonal functions in the given interval $\left[0, R_{0}\right]$. Hence, any field in the initial plane $z=0$ can be decomposed into these modal solutions.

Solutions (3) are propagation-invariant in free space, i.e., they are non-diffractive Bessel vector beams with radial and azimuthal indices possessing polarization and phase singularities. Note that the non-diffractive scalar Bessel beams in free space were proposed in [60]. In [61], three-dimensional self-imaging electromagnetic fields that are exact solu- 
tions of Maxwell's equations were analyzed. In [62], the amplitude components of the vector non-diffractive beams were obtained as solutions to the vector Helmholtz wave equation. It was shown in [63] that the normalized Bessel beams with radial indices are the solutions of the scalar Helmholtz equation.

Using Dirac notations, the vector hybrid modes (3) can be represented as:

$$
\left|\Psi_{p l}(r, \varphi, z)\right\rangle=\frac{1}{2}|p, l\rangle\left(|l, R\rangle e^{-i \varphi}+|-l, L\rangle e^{i \varphi}\right) e^{i \beta_{p l} z}
$$

where $|p, l\rangle=\psi_{p l}(r),|l, R\rangle=e^{i l \varphi+i \varphi_{0}}\left(\begin{array}{c}1 \\ -i\end{array}\right)$ and $|-l, L\rangle=e^{-i l \varphi+i \varphi_{0}}\left(\begin{array}{c}1 \\ i\end{array}\right)$.

When $l=0$, Equation (5) reduces to the electric field for the linearly polarized Bessel beams with non-zero intensity in the center. When $l=1$, Equation (5) describes the electric field of a radially or azimuthally polarized beam with annular intensity distribution. Light beams with radial and azimuthal polarizations are the most well-known cylindrical vector beams with phase (scalar) and polarization (vector) singularities. The phase singularity is associated with a phase (scalar) vortex of a beam or with the helical wavefront. The polarization singularity occurs in beams with spatially varying polarization distribution $[45,46]$.

Cartesian components of the field are related to polar by the relationship:

$$
\Psi_{p l}(r, \varphi, z)=\left(\begin{array}{c}
e_{x} \\
e_{y}
\end{array}\right)=\left(\begin{array}{cc}
\cos \varphi & -\sin \varphi \\
\sin \varphi & \cos \varphi
\end{array}\right)\left(\begin{array}{c}
e_{r} \\
e_{\varphi}
\end{array}\right)=\frac{1}{2}|p, l\rangle(|l, R\rangle+|-l, L\rangle) e^{i \beta_{p l} z}
$$

Thus, the vector mode represents the superposition of beams in orthogonal polarization (spin) and OAM states. A spin part is associated with polarization and an orbital part is associated with spatial distribution. There is an entanglement or non-separability between spatial, polarization and orbital angular momentum degrees of freedom.

Arbitrary incident beam at $z=0$ with spatial transverse dimensions significantly exceeding the wavelength can be decomposed in the series of modal solutions:

$$
\vec{E}(r, \varphi, 0)=\sum_{p l}\left(\begin{array}{c}
a_{p l}^{r} \\
a_{p l}^{\varphi}
\end{array}\right) \vec{e}(r, \varphi, 0),
$$

where

$$
\begin{aligned}
& a_{p l}^{r}=\left\langle e_{r}(r, \varphi, 0) \mid E_{r}(r, \varphi, 0)\right\rangle=\int_{0}^{\infty} \int_{0}^{2 \pi} E_{r}(\rho, 0) e_{r}^{*}(r, \varphi, 0) \rho d \rho d \varphi, \\
& a_{p l}^{\varphi}=\left\langle e_{\varphi}(r, \varphi, 0) \mid E_{\varphi}(r, \varphi, 0)\right\rangle=\int_{0}^{\infty} \int_{0}^{2 \pi} E_{\varphi}(\rho, 0) e_{\varphi}^{*}(r, \varphi, 0) \rho d \rho d \varphi,
\end{aligned}
$$

and

$$
\int_{0}^{\infty} \int_{0}^{2 \pi} E^{*}(\rho, 0) E(\rho, 0) \rho d \rho d \varphi=1
$$

The evolution of the incident field is determined by the expression

$$
\vec{E}(r, \varphi, z)=\sum_{p l}\left(\begin{array}{c}
a_{p l}^{r} \\
a_{p l}^{\varphi}
\end{array}\right) \Psi_{p l}(r, \varphi, 0) e^{i \beta_{p l} z}
$$

where $\beta_{p l}$ are the propagation constants of the modes.

\section{Degree of Polarization}

The degree of polarization is determined from the calculations of the coherency matrix [1]:

$$
\Phi=\left(\begin{array}{ll}
\left\langle E_{r}^{*} E_{r}\right\rangle & \left\langle E_{r}^{*} E_{\varphi}\right\rangle \\
\left\langle E_{\varphi}^{*} E_{r}\right\rangle & \left\langle E_{\varphi}^{*} E_{\varphi}\right\rangle
\end{array}\right)
$$


The angular brackets define the ensemble average taken over the statistical ensemble representing the randomly varying electromagnetic field. The degree of polarization is determined by the expression [1]:

$$
P=\left(1-\frac{4 \operatorname{det} \Phi}{S p^{2} \Phi}\right)^{1 / 2}
$$

where

$$
\begin{gathered}
\Phi_{i, j}=\left\langle e_{i}|\hat{\Phi}(r, z)| e_{j}\right\rangle, i, j=r, \varphi, \\
\Phi_{r r}(r, z)=\sum_{p p^{\prime}} A_{p l p^{\prime} l^{\prime}}^{r r} \Psi_{p l}^{r}(r, \varphi) \Psi_{p^{\prime} l^{\prime}}^{* r}(r, \varphi) \cos \left[\left(\beta_{p l}-\beta_{p^{\prime} l^{\prime}}\right) z\right], \\
\Phi_{r \varphi}(r, z)=\sum_{p p^{\prime}} A_{p l p^{\prime} l^{\prime}}^{r \varphi} \Psi_{p l}^{r}(r, \varphi) \Psi_{p^{\prime} l^{\prime}}^{* \varphi}(r, \varphi) \cos \left[\left(\beta_{p l}-\beta_{p^{\prime} l^{\prime}}\right) z\right], \\
\Phi_{\varphi r}(r, z)=\sum_{p p^{\prime}} A_{p l p^{\prime} l^{\prime}}^{\varphi r} \Psi_{p l}^{\varphi}(r, \varphi) \Psi_{p^{\prime} l^{\prime}}^{* r}(r, \varphi) \cos \left[\left(\beta_{p l}-\beta_{p^{\prime} l^{\prime}}\right) z\right], \\
\Phi_{\varphi \varphi}(r, z)=\sum_{p p^{\prime}} A_{p l p^{\prime} l^{\prime}}^{\varphi \varphi} \Psi_{p l}^{\varphi}(r, \varphi) \Psi_{p^{\prime} l^{\prime}}^{* \varphi}(r, \varphi) \cos \left[\left(\beta_{p l}-\beta_{p^{\prime} l^{\prime}}\right) z\right], \\
A_{p l p l l \prime}^{i j}=a_{p l}^{i} a_{p^{\prime} l^{\prime}}^{j} \ldots A_{p l p l^{\prime} l^{\prime}}^{r r}=a_{p p p^{\prime}}^{r r}=a_{p l}^{r} a_{p^{\prime} l^{\prime}}^{r} .
\end{gathered}
$$

In the experiments, the degree of polarization $P$ can be determined from the measurements of the Stokes parameters [1,2]:

$$
P=\left(S_{1}^{2}+S_{2}^{2}+S_{3}^{2}\right)^{1 / 2}
$$

where $S_{0}=I_{x}+I_{y}$ is the total intensity,

$$
\begin{gathered}
S_{1}=I_{x}-I_{y}, \\
S_{2}=I_{45^{\circ}}+I_{-45^{\circ}}, \\
S_{3}=I_{R C P}-I_{L C P} \\
S_{12}=S_{1}+i S_{2}=A_{12} \exp \left(i \phi_{12}\right),
\end{gathered}
$$

$\phi_{12}=\tan ^{-1}\left(S_{2} / S_{1}\right)$ is the Stokes phase.

Note that, at the polarization singularities, the Stokes phase $\phi_{12}$ is the undefined quantity [45].

There is an analogy between the coherency matrix and the quantum-mechanical density matrix [64]:

$$
\rho=\left(\begin{array}{ll}
\rho_{r r} & \rho_{r \varphi} \\
\rho_{\varphi r} & \rho_{\varphi \varphi}
\end{array}\right),
$$

where $\rho$ is the density matrix corresponding to the normalized coherence function $\Lambda_{2}=\Phi / \operatorname{Sp}(\Phi)$.

This analogy allows such thermodynamic parameters as entropy, temperature, etc. for the optical radiation to be introduced [64]. The entropy of the optical beam can be determined as

$$
S=-\sum_{n} w_{n} \ln w_{n}
$$

where $w_{n}=\frac{\langle n|\hat{\rho}| n\rangle}{S p \hat{\rho}}$ is the probability of excitation of a given mode, i.e., the fraction of radiation energy carried by this mode.

It is known that the entropy is the measure of the lack of information about the system. The entropy reaches its minimum $(S=0)$ in the case of totally polarized pure state and has a maximum $(S=\infty)$ for unpolarized and incoherent radiation.

The density matrix operator has a trace $S p \rho=1$ and for the mixed (partially polarized) state $S p \rho^{2} \leq 1$ 
The value $S p \rho^{2}$ is a measure of quantum-mechanical purity. The purity parameter or impurity can be introduced to describe the partially polarized beams [65]:

$$
\zeta=1-S p \rho^{2}
$$

where

$$
S p \rho^{2}=\sum_{p l, p / l,} \rho_{p l, p \prime l l} \rho_{p / l, p l},
$$

$\rho_{p l, p^{\prime} l^{\prime}}=\langle\Psi|\hat{\rho}| \Psi\rangle,\left\langle\Psi\left|=\Psi_{p^{\prime} l^{\prime}}^{*}(r, \varphi),\right| \Psi\right\rangle=\Psi_{p l}(r, \varphi), S p \rho=\sum_{p} \rho_{p p}=1$.

There is a close relationship between the degree of polarization and the quantum purity parameter of the light beam, so the degree of polarization can be represented by [65]:

$$
\left.P=\sqrt{1-2\left(1-S p\left(\hat{\rho}^{2}\right)\right.}\right)
$$

It follows from (15) that the trace of the matrix $\rho^{2}$ is given by the expression

$$
S p \rho^{2}=\frac{1}{2}\left(1+P^{2}\right)
$$

and, hence, the equality $S p \rho^{2}=1$ is the necessary and sufficient condition for finding a beam in a pure state.

The degree of polarization $P=1$ corresponds to a pure state and $P=0$ corresponds to an unpolarized beam. Note that we use here the term "purity" with respect to coherent fields. We show that the purity of a beam is preserved during propagation if only this beam is a polarization-invariant modal solution of Maxwell's equations.

\section{Simulation Results}

Consider the incident vector beam at $z=0$ with Gaussian and Bessel-Gauss (BG) spatial distributions of the intensity:

$$
\begin{gathered}
\vec{E}(r, \varphi, 0)=\sqrt{\frac{2}{\pi}} \frac{1}{a_{0}} \exp \left(-r^{2} / a_{0}^{2}\right)\left(\begin{array}{c}
\cos \varphi \\
-\sin \varphi
\end{array}\right), \\
\vec{E}(r, \varphi, 0)=A_{0} \exp \left(-r^{2} / w_{0}^{2}\right) J_{0}(\gamma r)\left(\begin{array}{c}
\cos \varphi \\
-\sin \varphi
\end{array}\right),
\end{gathered}
$$

where $a_{0}$ is the radius of a Gaussian beam, $A_{0}=\sqrt{\frac{2}{\pi}} \frac{1}{w_{0}} \exp \left(\frac{1}{8} \gamma^{2} w_{0}^{2}\right)\left[I_{0}\left(\frac{1}{4} \gamma^{2} w_{0}^{2}\right)\right]^{-1 / 2}$, $w_{B} / \mu_{1}=\gamma^{-1}$ is the effective width of the BG beam.

These beams are linearly polarized, so they can be represented by the decomposition into the modal solutions with zero OAM $(l=0)$. The modal amplitude coefficients for these incident beams can be calculated analytically. The expressions for modal coefficients have the form:

$$
\begin{gathered}
a_{p l}^{r}=a_{p l}^{\varphi}=\frac{\sqrt{2} a_{0}}{R_{0} J_{1}\left(\mu_{p}\right)} \exp \left\{-\frac{a_{0}^{2} \mu_{p}^{2}}{4 R_{0}^{2}}\right\}, \\
a_{p l}^{r}=a_{p l}^{\varphi}=\frac{A_{0} \sqrt{\pi} w_{0}^{2}}{R_{0} J_{1}\left(\mu_{p}\right)} \exp \left[-\left(\alpha^{2}+\gamma^{2}\right) w_{0}^{2} / 4\right] I_{0}\left(\frac{\alpha \gamma}{2} w_{0}^{2}\right),
\end{gathered}
$$

where $\mu_{p}$ are the positive zeros of the Bessel function $J_{0}(z)$, and $I_{0}(z)$ is the modified Bessel function of the first kind.

Note that polarization-invariant linear and circular polarized vector beams have no vortices $(l=0)$, i.e., such beams have non-zero intensity on the axis and no OAM. On the contrary, polarization-invariant radially and azimuthally polarized vector beams have non-zero angular orbital momentum and annular intensity distributions. 


\subsection{Purity}

The quantum-mechanical purity of a beam can be calculated using (14). Initial density matrix operator for a pure state is given by the incident vector beams: $\rho=|\vec{E}\rangle\langle\vec{E}|$.

\section{Purity Parameter}

In Figure 1, the dependences of the quantum-mechanical purity $S p \rho^{2}$ and the purity parameter (impurity) $\zeta=1-S p \rho^{2}$ on the propagation distance are presented for different widths of linearly polarized Gaussian beam with zero OAM. Figure $1 \mathrm{~b}$ shows the dependence of the quantum purity parameter of the beam on the distance. It can be seen that if the beam is in a pure state in the initial plane, then, during propagation, the beam transforms into a mixed state.

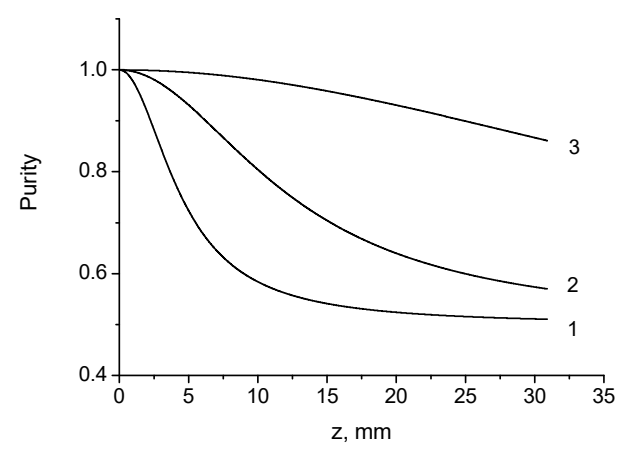

(a)

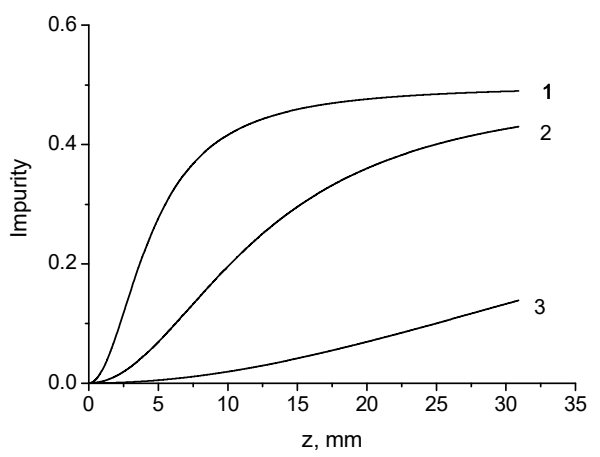

(b)

Figure 1. The purity (a) and impurity (b) as function of distance. $1-a_{0}=30 \mu \mathrm{m} ; 2-a_{0}=50 \mu \mathrm{m}$; $3-a_{0}=100 \mu \mathrm{m} . \lambda=0.63 \mu \mathrm{m}$.

In Figure 2a, the dependence of the impurity on distance is shown for a beam radius $a_{0}=15 \mu \mathrm{m}$. In Figure $2 \mathrm{~b}$, the dependence of an entropy of a Gaussian beam as function of propagation distance is shown. It can be seen that there is a significant change in entropy at a length of the order of the diffraction length.

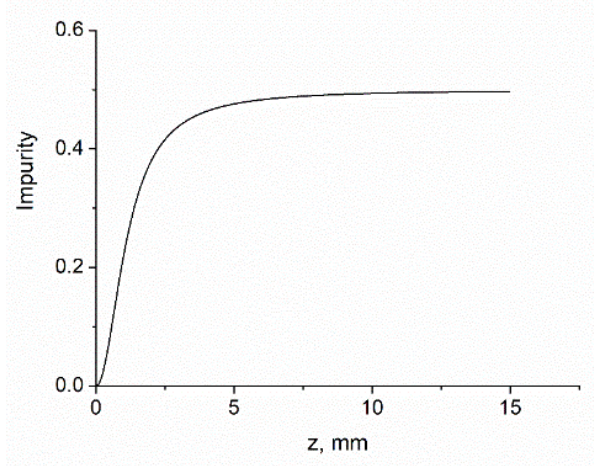

(a)

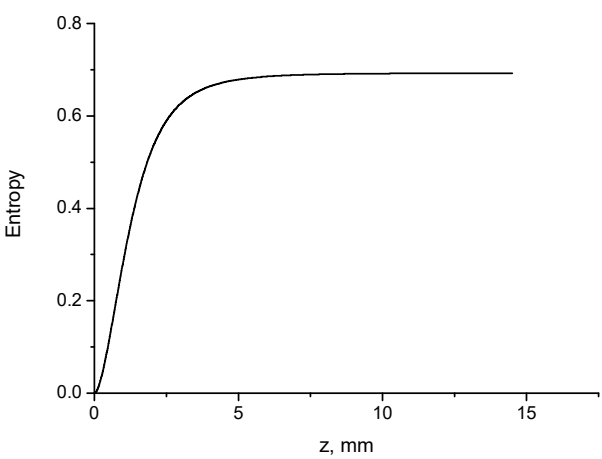

(b)

Figure 2. (a) Impurity as function of distance. (b) Entropy as function of distance. $a_{0}=15 \mu \mathrm{m}$.

\subsection{Degree of Polarization}

\subsubsection{Linear Polarization}

The degree of polarization can be calculated using the expression (15). Figure 3a shows the change in the degree of polarization of linearly polarized Gaussian light beams with a distance for different beam radii. It can be seen from the figure that a strong change in the degree of polarization occurs at distances of the order of the diffraction length $l_{d}=k a_{0}^{2} / 2$. This means that the focused beams are depolarized at a shorter distance. Figure $3 \mathrm{~b}$ shows 
the change in the degree of polarization of a linearly polarized Gaussian light beam with a distance for the beam radius $a_{0}=15 \mathrm{~mm}$.

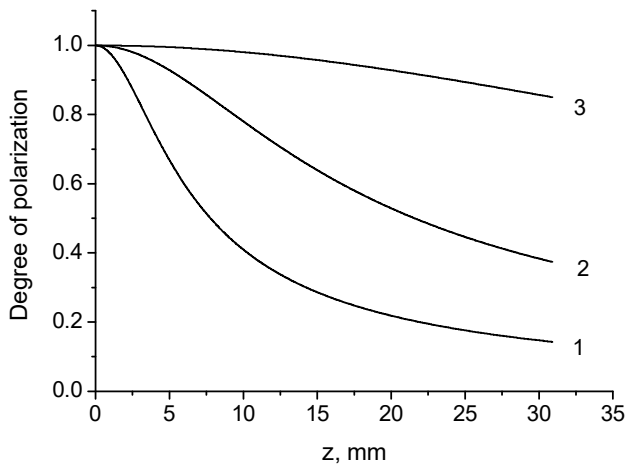

(a)

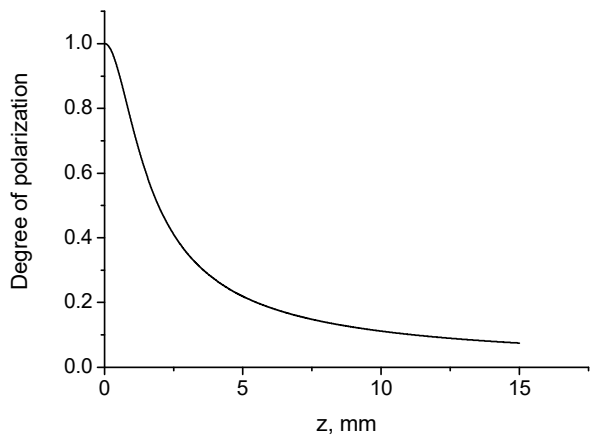

(b)

Figure 3. (a) Degree of polarization as function of distance. $1-a_{0}=30 \mu \mathrm{m} ; 2-a_{0}=50 \mu \mathrm{m}$; $3-a_{0}=100 \mu \mathrm{m}$. (b) Degree of polarization as function of distance. $a_{0}=15 \mu \mathrm{m}$.

Note that a coherence-induced decrease in the degree of polarization with the propagation distance for partially coherent beams in free space was shown in $[19,25,26]$. It was found that for a completely spatially coherent beam, its degree of polarization does not change during propagation $[19,25,26]$. The spectral degree of polarization remains propagation-invariant along the axis of a Gaussian Shell-model beam [27,28]. Here, we show that the decrease in the degree of polarization occurs also for spatially coherent beams. Unlike the coherence-induced mechanism of polarization changes, the decrease in the degree of polarization with distance arises also due to the interference between the modes having different propagation constants. A decrease in the degree of correlation between different modes takes place due to a difference in propagation velocities of Bessel vector modes with different radial indices.

Note that for very small radii of the incident beam $\left(a_{0}<<\lambda\right)$, the two-dimensional description of polarization is incomplete. It was shown in $[66,67]$ that knowledge of the vector polarization of radiation alone is not sufficient to fully determine the polarization state of a focused light beam. Additional parameters (tensor polarization components) should be taken into account. It is shown in [66] that the vector and tensor degrees of polarization are sufficient for a complete description of the three-component field of a light beam. In Figure 4, the dependences of the degree of polarization on the propagation distance are shown for Gaussian and BG beams with approximately similar effective widths.

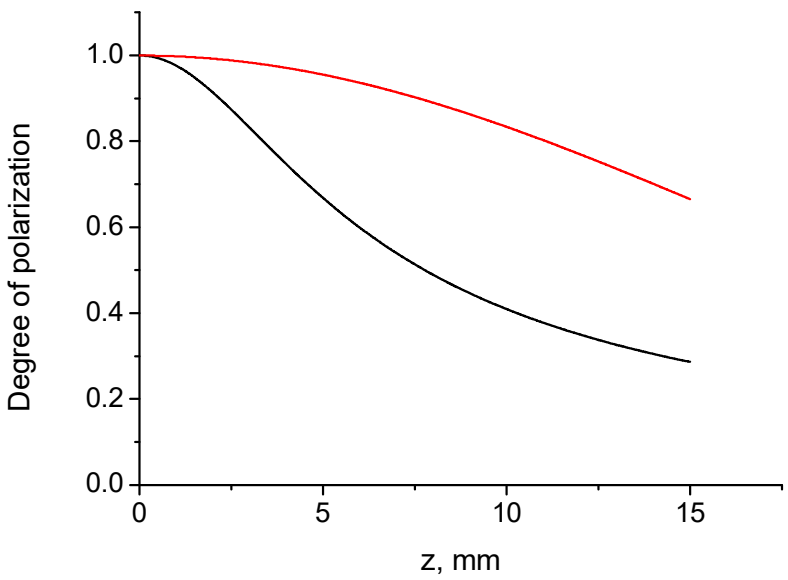

Figure 4. Degree of polarization as function of distance. Gaussian beam (black line), $a_{0}=30 \mu \mathrm{m}$; BG beam (red line), $w_{0}=200 \mu \mathrm{m}, w_{B}=40 \mu \mathrm{m}$. 
It can be seen that a faster decrease in the degree of polarization occurs for the Gaussian beam (17). The degree of polarization of the BG beam (18) decreases more slowly than for a Gaussian beam if $w_{0} \gg w_{B}$. Indeed, in this case, the BG beam becomes more identical to the Bessel beam. The intensity profiles of the incident Gaussian (17) and Bessel-Gauss (18) beams are shown in Figure 5.

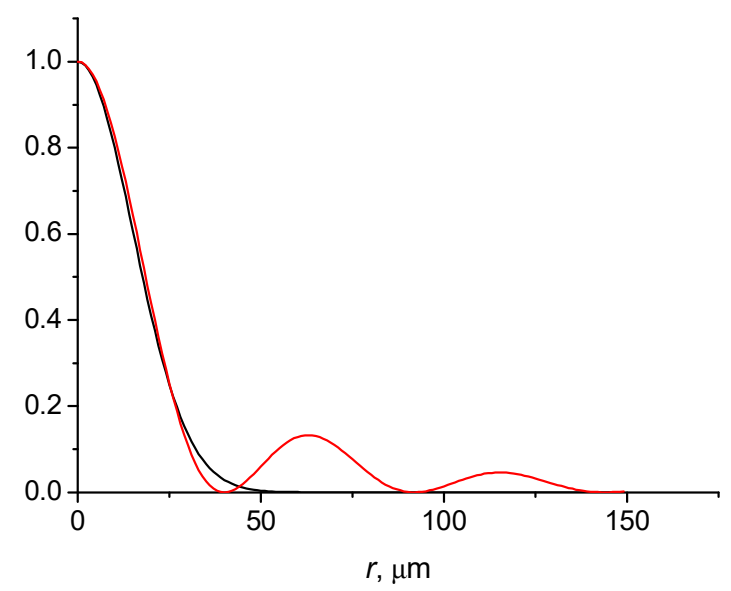

Figure 5. Intensity profiles of Gaussian (black line) and BG (red line) beams. $a_{0}=30 \mu \mathrm{m}, w_{0}=200 \mu \mathrm{m}$, $w_{B}=40 \mu \mathrm{m}$.

\subsubsection{Radial Polarization}

Consider the radially polarized incident vector beams at $\mathrm{z}=0$ with annular Gaussian and Bessel-Gauss spatial distributions of the intensity and OAM $1=1$ :

$$
\begin{gathered}
\vec{E}(r, \varphi, 0)=\frac{2}{\sqrt{\pi}} \frac{r}{a_{0}^{2}} \exp \left(-r^{2} / a_{0}^{2}\right)\left(\begin{array}{l}
1 \\
0
\end{array}\right), \\
\vec{E}(r, \varphi, 0)=A_{0} \exp \left(-r^{2} / w_{0}^{2}\right) J_{1}(\gamma r)\left(\begin{array}{l}
1 \\
0
\end{array}\right),
\end{gathered}
$$

where $a_{0}$ is the radius of a Gaussian beam, $A_{0}=\sqrt{\frac{2}{\pi}} \frac{1}{w_{0}} \exp \left(\frac{1}{8} \gamma^{2} w_{0}^{2}\right)\left[I_{1}\left(\frac{1}{4} \gamma^{2} w_{0}^{2}\right)\right]^{-1 / 2}$, and $w_{B} / \mu_{1}=\gamma^{-1}$ is the effective width of the BG beam.

For the modal coefficients we have

$$
\begin{gathered}
a_{p l}^{r}=a_{p l}^{\varphi}=\frac{a_{0}^{2}}{R_{0}^{2}} \frac{\mu_{p}}{J_{2}\left(\mu_{p}\right)} \exp \left\{-\frac{a_{0}^{2} \mu_{p}^{2}}{4 R_{0}^{2}}\right\}, \\
a_{p l}^{r}=a_{p l}^{\varphi}=\frac{A_{0} \sqrt{\pi} w_{0}^{2}}{R_{0} J_{2}\left(\mu_{p}\right)} \exp \left[-\left(\alpha^{2}+\gamma^{2}\right) w_{0}^{2} / 4\right] I_{1}\left(\frac{\alpha \gamma}{2} w_{0}^{2}\right),
\end{gathered}
$$

where $\mu_{p}$ are the positive zeros of the Bessel function $J_{1}(z)$, and $I_{1}(z)$ is the modified Bessel function of the first kind.

In Figure 6a, the dependences of the degree of polarization on the propagation distance are presented for the beams with annular Gaussian intensity distribution (21) and $\mathrm{OAM} 1=1$.

It can be seen that the smaller the radius of the beam, the smaller the distance at which the noticeable depolarization of the beam occurs. In Figure $6 \mathrm{~b}$, the degrees of polarization as function of distance are presented for Gaussian (21) and BG (22) beams with OAM $1=1$ and with approximately similar effective widths (Figure 7).

It follows that the decrease in the degree of polarization of the BG beam (22) occurs much slower than for a Gaussian beam (21) if the value $w_{0}$ exceeds the effective radius of the BG beam $w_{B}$. In this case, the behavior of the BG beam is similar to the vector Bessel 
beam, which is a modal solution. The closer the beam is to the modal solution, the weaker the beam depolarization with distance.

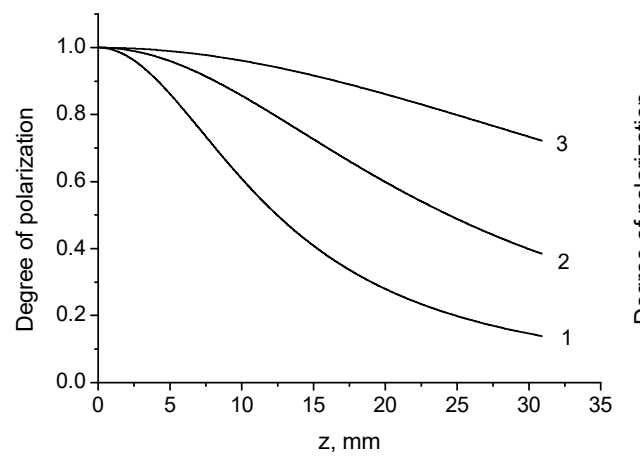

(a)

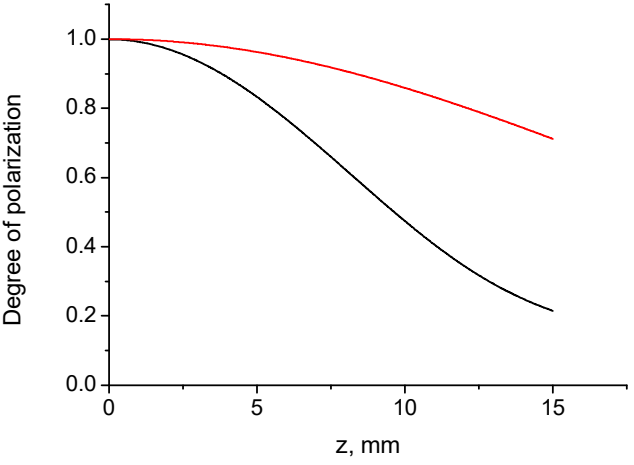

(b)

Figure 6. (a) Degree of polarization as function of distance. $1-a_{0}=50 \mu \mathrm{m} ; 2-a_{0}=70 \mu \mathrm{m}$; $3-a_{0}=100 \mu \mathrm{m} . \lambda=0.63 \mu \mathrm{m}$. (b) Degree of polarization as function of distance. Gaussian beam (black line), $a_{0}=45 \mu \mathrm{m}$; BG beam (red line), $w_{B}=70 \mu \mathrm{m} ; w_{0}=200 \mu \mathrm{m} . \lambda=0.63 \mu \mathrm{m}$.

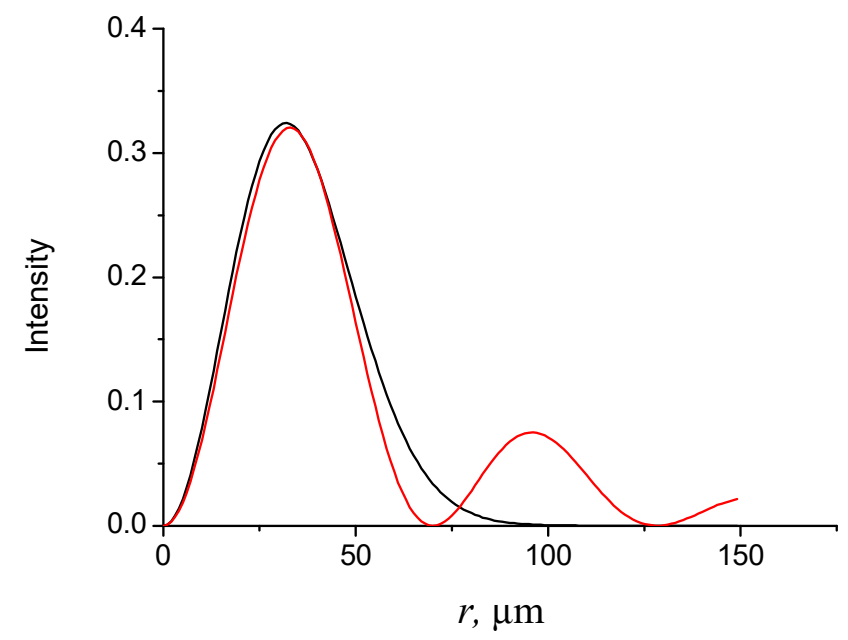

Figure 7. Intensity profiles of Gaussian (black line) and BG (red line) beams. $a_{0}=45 \mu \mathrm{m} ; w_{0}=200 \mu \mathrm{m}$, $w_{B}=70 \mu \mathrm{m}$.

It should be noted that the simulation results presented above correspond to the degree of polarization, which is averaged over the cross section of the beam. The values of the local degree of polarization determined at a given point of the transverse plane are usually considered. It is known that the local degree of polarization varies with displacement from the axis of the beam, i.e., the polarization is inhomogeneous in cross section [19]. However, the average degree of polarization is usually determined during measurements.

\section{Discussion}

Thus, polarization-invariant hybrid vector Bessel beams with phase and polarization singularities are proposed, which combine spin and OAM and are the solutions of Maxwell's equations in free space. These modal solutions with discrete azimuthal and radial indices form a complete set of mutually orthogonal functions. Consequently, an arbitrary vector beam in the initial plane $z=0$ can be decomposed into these modal solutions, the evolution of which is determined by the propagation constant $\beta_{p l}$. The modal approach provides clear physical insight into the depolarization mechanism of vector beams in free space and computational simplification in the analysis. 
One of the important properties of hybrid vector beams, which are the modal solutions, is the entanglement of phase and spin degrees of freedom describing the orbital angular momentum and the polarization vector in orthogonal basis vectors of circular polarization. These complex modes cannot simply be expressed through the TE and TM modes. The entanglement property is inherent in quantum systems and may have implications for future quantum networks [68-71]. In [70], it was demonstrated that the entanglement, or non-separability, between the spatial and polarization degrees of freedom also experiences self-healing.

Hybrid vector modes have non-separable spatial, phase and polarization degrees of freedom. Hence, there is an analogy between the non-separability of such modes and entanglement of quantum states. Here, we found that quantum-mechanical purity is a measure of the average degree of polarization. In turn, the purity is related to the entropy.

Note that the vector beam with separable spin and OAM degrees of freedom in the initial plane $\mathrm{z}=0$ transforms into the polarization-OAM entanglement beam during propagation [72,73].

Degree of polarization of pure states, which are the modal solutions of the Maxwell equations, remains invariant during propagation, i.e., it does not change on propagation. This indicates that the purity parameter and entropy for the modal solutions remain unchanged. A decrease in the degree of polarization with distance arises due to the interference between the modes having different propagation constants. Due to differences in propagation velocities of Bessel vector modes with different radial indices, a decrease in the degree of correlation between the different modes takes place. The more propagating modes are excited, the more the degree of polarization decreases with distance.

Note, it was shown in [24-28], that the change in the degree of polarization of the beam is influenced by the coherence properties of the field in the source plane. Here, we have demonstrated that, unlike the depolarization of partially coherent beams, the depolarization of coherent beams occurs due to interference between propagating modes.

Here we considered $2 \times 2$ matrices to describe the polarization of vector beams. Indeed, in conventional paraxial optics, the fields are transverse, so the polarization is well described by Stokes parameters determined from the $2 \times 2$ coherency matrix [1-3]. Although the electromagnetic field in the far zone is transverse, many problems of near-field optics, data storage, optical microscopy, and focusing and scattering need to be analyzed, taking into account the longitudinal component of the field $E_{\mathrm{z}}$. For strongly focused beams, the Stokes $2 \times 2$ formalism should be extended to $3 \times 3$ coherency matrices [74-79]. In $[67,72,73]$, the evolution of three-dimensional electromagnetic fields in a graded-index medium was studied. The polarization state of the most general two-dimensional field can be completely described by three parameters. This is related to as vector polarization. For a three-dimensional field except for 3 components of the vector polarization, there are 5 components of tensor polarization. The complexity of 3D electromagnetic field arises from the tensor polarization [66,67]. In contrast to the 2D case, where any beam can be represented as the superposition of totally polarized and unpolarized light, in the 3D case, an arbitrary beam cannot be represented as a mixture of an unpolarized beam (one parameter) and a polarized beam (5 parameters) because nine independent parameters are needed to characterize the coherence matrix.

We have considered the polarization properties of coherent vector beams. Analysis of the propagation of partially polarized and partially coherent beams is important for elucidating the physical mechanisms of depolarization. Future research will be related to polarization effects of partially coherent vector vortex beams [80-84]. Of particular interest is the study of the effects of non-paraxiality in the propagation of focused vector beams [85-98], of evanescent waves [99,100] and the effects of coherence and polarization of pulsed beams [101]. Other important topics are the polarization effects in plasmonic structures, including subwavelength gratings [102], multilayer devices and microlens arrays for imaging systems [103-108], diffractive optical elements [109-111], etc. New 
directions in technology are opening up thanks to the possibilities of controlling the spin angular momentum and Berry phase [112,113].

Recently, new developments based on the transverse spin phenomenon, which occurs in focused fields, have been of great interest [114-118]. New optical phenomena associated with the angular momentum have been demonstrated in [119]. It was shown in [115] that transverse spin manifests itself even in completely unpolarized fields. There is a connection between 3D polarization in the transverse spin [117] and non-paraxial fields [118] owing to the spin-orbit interaction of light. These phenomena can be used in telecommunications, nanoelectronics, optical imaging, etc. [119,120].

\section{Conclusions}

In summary, new hybrid vector Bessel beams with polarization-OAM entanglement, which are the modal solutions of the Maxwell equations, are proposed to study the evolution of vector beams in free space. Non-separability of degrees of freedom associated with the spatial coordinates, spin (polarization) and OAM (phase) is the main feature of the vector vortex beams which are the modal solutions.

The vector mode decomposition method was developed for the analysis of the evolution of the polarization of arbitrary transverse incident beams with different spatial intensity distributions. Using the decomposition of the field of an arbitrary incident vector beam into the hybrid modal solutions, the change in the 2D degree of polarization, the quantum purity parameter and the entropy of the beam with distance was investigated.

The change in the degree of polarization of vector vortex beams on propagation in free space due to interference between different propagating modes has been demonstrated. A significant decrease in the degree of polarization occurs at the diffraction length $l_{\mathrm{d}}$ for incident beams that are not vector modal solutions.

Close connection of the degree of polarization with the quantum-mechanical purity parameter is emphasized.

The results obtained may be useful in free-space optical communications, singular optics, photonics, optical coherence tomography, imaging, and may have implications for future quantum networks.

Funding: This research was funded by the Ministry of Science and Higher Education of the Russian Federation under the State contract FFNS-2022-0009 and by the Russian Foundation for Basic Research, project number 19-29-11026.

Conflicts of Interest: The author declares no conflict of interest.

\section{References}

1. Wolf, E. Introduction to the Theory of Coherence and Polarization of Light; Cambridge University Press: Cambridge, UK, 2007.

2. Azzam, R.; Bashara, N. Ellipsometry and Polarized Light; North-Holland Publishing Company: New York, NY, USA, 1977.

3. Chipman, R.; Lam, W.; Young, G. Polarized Light and Optical Systems; CRC Press: Boca Raton, FL, USA, 2018.

4. Tatarskii, V.I. Estimation of light depolarization by turbulent inhomogeneities of the atmosphere. Izv. VUZov Radiofizika 1967, 10, 1762-1765.

5. Kravtzov, I.; Yu, A. Geometrical depolarization of light in a turbulent atmosphere. Izv. VUZov Radiofizika 1970, 13, $281-284$.

6. Rytov, S.M. On transition from wave to geometrical optics. Dokl. Akad. Nauk USSR 1938, 18, 263-266.

7. Vladimirsky, V.V. On rotation of polarization plane in twisted ray of light. Dokl. Akad. Nauk USSR 1941, 31, $222-225$.

8. Cohen, L.G. Measured attenuation and depolarization of light transmitted along glass fibers. Bell Syst. Tech. J. 1971, 50, 23-42. [CrossRef]

9. Smith, A.M. Polarization and magnetooptic properties of single-mode optical fiber. Appl. Opt. 1978, 17, 52-56. [CrossRef]

10. Kaminov, I.P. Polarization in optical fibers. IEEE J. Quantum Electron. 1981, 17, 15-22. [CrossRef]

11. Shatrov, A.D. Polarization effects in multimode graded-index fibers. Radiotekh. Elektr. 1988, 26, 505-512.

12. Esayan, A.A.; Zel'dovich, B.Y. Depolarization of radiation in an ideal multimode graded-index optical fiber. Sov. J. Quantum Electron. 1988, 18, 149-150. [CrossRef]

13. Kotov, O.I.; Marusov, O.L.; Nikolaev, O.L.; Filippov, V.N. Polarization properties of optical fibers. Modal approach. Opt. Spectr. 1991, 70, 924-927.

14. Petrov, N.I. Depolarization of light in a graded-index isotropic medium. J. Mod. Opt. 1996, 43, 2239-2249. [CrossRef]

15. Petrov, N.I. Evolution of polarization in an inhomogeneous isotropic medium. JETP 1997, 85, 1085-1093. [CrossRef] 
16. Petrov, N.I. Depolarization of Light in Optical Fibers: Effects of Diffraction and Spin-Orbit Interaction. Fibers 2021, 9, 34. [CrossRef]

17. Zel'dovich, B.Y.; Kundikova, N.D. Intrafibre rotation of the plane of polarization. Quantum Electr. 1995, 25, 172-174. [CrossRef]

18. James, D.F.V. Change of polarization of light beams on propagation in free space. J. Opt. Soc. Am. A 1994, 11, 1641-1643. [CrossRef]

19. Agrawal, G.P.; Wolf, E. Propagation-induced polarization changes in partially coherent optical beams. J. Opt. Soc. Am. A 2000, 17, 2019-2023. [CrossRef]

20. Korotkova, O.; Wolf, E. Changes in the state of polarization of a random electromagnetic beam on propagation. Opt. Commun. 2005, 246, 35-43. [CrossRef]

21. Korotkova, O.; Hoover, B.G.; Gamiz, V.L.; Wolf, E. Coherence and polarization properties of far fields generated by quasihomogeneous planar electromagnetic sources. J. Opt. Soc. Am. A 2005, 22, 2547-2556. [CrossRef]

22. Korotkova, O.; Visser, T.D.; Wolf, E. Polarization properties of stochastic electromagnetic beams. Opt. Commun. 2008, 281, 515-520. [CrossRef]

23. Ellis, J.; Dogariu, A.; Ponomarenko, S.; Wolf, E. Degree of polarization of statistically stationary electromagnetic fields. Opt. Commun. 2005, 248, 333-337. [CrossRef]

24. Salem, M.; Wolf, E. Coherence-induced polarization changes in light beams. Opt. Lett. 2008, 33, 1180-1182. [CrossRef] [PubMed]

25. Vidal, I.; Fonseca, E.J.S.; Hickmann, J.M. Light polarization control during free-space propagation using coherence. Phys. Rev. A 2011, 84, 033836. [CrossRef]

26. Wu, G.; Wang, F.; Cai, Y. Coherence and polarization properties of a radially polarized beam with variable spatial coherence. Opt. Express 2012, 20, 28301-28318. [CrossRef] [PubMed]

27. Wolf, E. Polarization invariance in beam propagation. Opt. Lett. 2007, 32, 3400-3401. [CrossRef] [PubMed]

28. Zhao, D.; Wolf, E. Light beams whose degree of polarization does not change on propagation. Opt. Commun. 2008, 281, 3067-3070. [CrossRef]

29. Gori, F. Polarization basis for vortex beams. J. Opt. Soc. Am. A 2001, 18, 1612-1617. [CrossRef]

30. Martınez-Herrero, R.; Mejıas, P.M. Propagation of light fields with radial or azimuthal polarization distribution at a transverse plane. Opt. Express 2008, 16, 9021-9033. [CrossRef]

31. Zhan, Q. Cylindrical vector beams: From mathematical concepts to applications. Adv. Opt. Photonics 2009, 1, 1-57. [CrossRef]

32. Santarsiero, M.; de Sande, J.C.G.; Piquero, G.; Gori, F. Coherence-polarization properties of fields radiated from transversely periodic electromagnetic sources. J. Opt. 2013, 15, 055701. [CrossRef]

33. Kotlyar, V.V.; Stafeev, S.S.; Nalimov, A.G. Sharp Focusing of a Hybrid Vector Beam with a Polarization Singularity. Photonics 2021, 8, 227. [CrossRef]

34. Khonina, S.N.; Porfirev, A.P.; Volotovskiy, S.G.; Ustinov, A.V.; Fomchenkov, S.A.; Pavelyev, V.S.; Schröter, S.; Duparré, M. Generation of Multiple Vector Optical Bottle Beams. Photonics 2021, 8, 218. [CrossRef]

35. Pääkkönen, P.; Tervo, J.; Vahimaa, P.; Turunen, J.; Gori, F. General vectorial decomposition of electromagnetic fields with application to propagation-invariant and rotating fields. Opt. Express 2002, 10, 949-959. [CrossRef] [PubMed]

36. Korotkova, O. Sufficient condition for polarization invariance of beams generated by quasi-homogeneous sources. Opt. Lett. 2011, 36, 3768-3770. [CrossRef] [PubMed]

37. Martínez-Herrero, R.; Mejías, P.M. Electromagnetic fields that remain totally polarized under propagation. Opt. Commun. 2007, 279, 20-22. [CrossRef]

38. de Sande, J.C.G.; Piquero, G.; Suárez-Bermejo, J.C.; Santarsiero, M. Mueller Matrix Polarimetry with Invariant Polarization Pattern Beams. Photonics 2021, 8, 491. [CrossRef]

39. Philip, G.M.; Viswanathan, N.K. Generation of spirally polarized propagation-invariant beam using fiber microaxicon. Opt. Lett. 2011, 36, 3906-3908. [CrossRef]

40. Alieva, T.; Rodrigo, J.A.; Camara, A.; Abramochkin, E. Partially coherent stable and spiral beams. J. Opt. Soc. Am. A 2013, 30, 2237-2243. [CrossRef]

41. Freund, I. Polarization singularities in optical lattices. Opt. Lett. 2004, 29, 875-877. [CrossRef]

42. Freund, I. Ordinary polarization singularities in three-dimensional optical fields. Opt. Lett. 2012, 37, 2223-2225. [CrossRef]

43. Khonina, S.N. Vortex beams with high-order cylindrical polarization: Features of focal distributions. Appl. Phys. B 2019, 125, 100. [CrossRef]

44. Jia, J.; Zhang, K.; Hu, G.; Hu, M.; Tong, T.; Mu, Q.; Gao, H.; Li, F.; Qiu, C.-W.; Zhang, P. Arbitrary cylindrical vector beam generation enabled by polarization-selective Gouy phase shifter. Photonics Res. 2021, 9, 1048-1054. [CrossRef]

45. Ruchi; Senthilkumaran, P.; Pal, S.K. Phase Singularities to Polarization Singularities. Int. J. Opt. 2020, 2020, 2812803. [CrossRef]

46. Wang, Q.; Tu, C.H.; Li, Y.N.; Wang, H.T. Polarization singularities: Progress, fundamental physics, and prospects. APL Photonics 2021, 6, 040901. [CrossRef]

47. Tovar, A.A. Production and propagation of cylindrically polarized Laguerre-Gaussian laser beams. J. Opt. Soc. Am. A 1998, 15, 2705-2711. [CrossRef]

48. Deng, D. Nonparaxial propagation of radially polarized light beams. J. Opt. Soc. Am. B 2006, 23, 1228-1234. [CrossRef]

49. Milione, G.; Sztul, H.I.; Nolan, D.A.; Alfano, R.R. Higher-order Poincaré sphere, Stokes parameters, and the angular momentum of light. Phys. Rev. Lett. 2011, 107, 053601. [CrossRef] 
50. Chen, S.; Zhou, X.; Liu, Y.; Ling, X.; Luo, H.; Wen, S. Generation of arbitrary cylindrical vector beams on the higher order Poincaré sphere. Opt. Lett. 2014, 39, 5274-5276. [CrossRef]

51. Liu, Z.; Liu, Y.; Ke, Y.; Liu, Y.; Shu, W.; Luo, H.; Wen, S. Generation of arbitrary vector vortex beams on hybrid-order Poincaré sphere. Photonics Res. 2017, 5, 15-21. [CrossRef]

52. Liu, W.; Liu, W.; Shi, L.; Kivshar, Y. Topological polarization singularities in metaphotonics. Nanophotonics 2021, 10, 1469-1486. [CrossRef]

53. He, C.; He, H.; Chang, J.; Chen, B.; Ma, H.; Booth, M.J. Polarization optics for biomedical and clinical applications: A review. Light Sci. Appl. 2021, 10, 194. [CrossRef]

54. Lim, S.W.D.; Park, J.S.; Meretska, M.L.; Dorrah, A.H.; Capasso, F. Engineering phase and polarization singularity sheets. Nat. Commun. 2021, 12, 4190.

55. Zhang, Y.B.; Liu, H.; Cheng, H.; Tian, J.G.; Chen, S.Q. Multidimensional manipulation of wave fields based on artificial microstructures. Opto-Electron. Adv. 2020, 3, 200002. [CrossRef]

56. Guo, Y.; Zhang, S.; Pu, M.; He, Q.; Jin, J.; Xu, M.; Zhang, Y.; Gao, P.; Luo, X. Spin-decoupled metasurface for simultaneous detection of spin and orbital angular momenta via momentum transformation. Light Sci. Appl. 2021, 10, 63. [CrossRef] [PubMed]

57. Gori, F. Matrix treatment for partially polarized, partially coherent beams. Opt. Lett. 1998, 23, 241-243. [CrossRef]

58. Wolf, E. Unified theory of coherence and polarization of random electromagnetic beams. Phys. Lett. A 2003, 312, $263-267$. [CrossRef]

59. Korn, G.A.; Korn, T.M. Mathematical Handbook; McGraw-Hill Book Company: New York, NY, USA, 1968.

60. Durnin, J. Exact solutions for nondiffracting beams. I. The scalar theory. J. Opt. Soc. Am. 1987, 4, 651-654. [CrossRef]

61. Turunen, J.; Friberg, A.T. Self-imaging and propagation-invariance in electromagnetic fields. Pure Appl. Opt. 1993, 2, 51-60. [CrossRef]

62. Bouchal, Z.; Olivik, M. Non-diffractive vector Bessel beams. J. Mod. Opt. 1995, 42, 1555-1566. [CrossRef]

63. Petrov, N.I. Speed of structured light pulses in free space. Sci. Rep. 2019, 9, 18332. [CrossRef]

64. Krivoshlykov, S.G.; Petrov, N.I.; Sisakyan, I.N. Density-matrix formalism for partially coherent optical fields propagating in slightly inhomogeneous media. Opt. Quantum Electr. 1986, 18, 253-264. [CrossRef]

65. Moya-Cessa, H.; Moya-Cessa, J.R.; Landgrave, J.E.A.; Martinez-Niconoff, G.; Perez-Leija, A.; Friberg, A.T. Degree of polarization and quantum-mechanical purity. J. Eur. Opt. Soc. Rapid Publ. 2008, 3, 08014. [CrossRef]

66. Petrov, N.I. Vector and Tensor Polarizations of Light Beams. Laser Phys. 2008, 18, 522-525. [CrossRef]

67. Petrov, N.I. Spin-orbit and tensor interactions of light in inhomogeneous isotropic media. Phys. Rev. A 2013, 88, 023815. [CrossRef]

68. Qian, X.F.; Eberly, J.H. Entanglement and classical polarization states. Opt. Lett. 2011, 36, 4110-4112. [CrossRef] [PubMed]

69. Otte, E.; Rosales-Guzmán, C.; Ndagano, B.; Denz, C.; Forbes, A. Entanglement beating in free space through spin-orbit coupling. Light Sci. Appl. 2018, 7, 18009. [CrossRef]

70. Otte, E.; Nape, I.; Rosales-Guzmán, C.; Valles, A.; Denz, C.; Forbes, A. Recovery of nonseparability in self-healing vector Bessel beams. Phys. Rev. A 2018, 98, 053818. [CrossRef]

71. Aiello, A.; Toppel, F.; Marquardt, C.; Giacobino, E.; Leuchs, G. Quantum-like nonseparable structures in optical beams. New J Phys. 2015, 17, 043024. [CrossRef]

72. Petrov, N.I. Vector Laguerre-Gauss beams with polarization-orbital angular momentum entanglement in a graded-index medium. J. Opt. Soc. Am. A 2016, 33, 1363-1369. [CrossRef]

73. Petrov, N.I. Splitting of levels in a cylindrical dielectric waveguide. Opt. Lett. 2013, 38, 2020-2022. [CrossRef]

74. Carozzi, T.; Karlsson, R.; Bergman, J. Parameters characterizing electromagnetic wave polarization. Phys. Rev. E 2000, 61, 2024-2028. [CrossRef]

75. Setälä, T.; Shevchenko, A.; Kaivola, M.; Friberg, A.T. Degree of polarization for optical near fields. Phys. Rev. E 2002, 66, 016615. [CrossRef] [PubMed]

76. Lindfors, K.; Setälä, T.; Kaivola, M.; Friberg, A.T. Degree of polarization in tightly focused optical fields. J. Opt. Soc. Am. A 2005, 22, 561-568. [CrossRef] [PubMed]

77. Sheppard, C.J.R. Jones and Stokes parameters for polarization in three dimensions. Phys. Rev. A 2014, 90, 023809. [CrossRef]

78. Sheppard, C.J.R. Partial polarization in three dimensions. J. Opt. Soc. Am. A 2011, 28, 1938-1947. [CrossRef]

79. Gil, J.J. Geometric Interpretation and General Classification of Three-Dimensional Polarization States through the Intrinsic Stokes Parameters. Photonics 2021, 8, 315. [CrossRef]

80. $\mathrm{Wu}, \mathrm{G}$. Propagation properties of a radially polarized partially coherent twisted beam in free space. J. Opt. Soc. Am. A 2016, 33, 345-350. [CrossRef]

81. Zhao, X.; Visser, T.D.; Agrawal, G.P. Controlling the degree of polarization of partially coherent electromagnetic beams with lenses. Opt. Lett. 2018, 43, 2344-2347. [CrossRef]

82. Ostrovsky, A.S.; García-García, J.; Rickenstorff-Parrao, C.; Olvera-Santamaria, M.A. Partially coherent diffraction-free vortex beams with a Bessel-mode structure. Opt Lett. 2017, 42, 5182-5185. [CrossRef]

83. Krivoshlykov, S.G.; Petrov, N.I.; Sisakyan, I.N. Spatial coherence of optical fields in longitudinally inhomogeneous media with the square-law index profile. Sov. J. Quantum Electron. 1985, 15, 330-338. [CrossRef]

84. Joshi, S.; Khan, S.N.; Manisha; Senthilkumaran, P.; Kanseri, B. Coherence-induced polarization effects in vector vortex beams. Opt. Lett. 2020, 45, 4815-4818. [CrossRef] 
85. Dong, Y.; Feng, F.; Chen, Y.; Zhao, C.; Cai, Y. Statistical properties of a nonparaxial cylindrical vector partially coherent field in free space. Opt. Express 2012, 20, 15908-15927. [CrossRef] [PubMed]

86. Yuan, Y.; Du, S.; Dong, Y.; Wang, F.; Zhao, C.; Cai, Y. Nonparaxial propagation properties of a vector partially coherent dark hollow beam. J. Opt. Soc. Am. A 2013, 30, 1358-1372. [CrossRef] [PubMed]

87. Gu, B.; Cui, Y. Nonparaxial and paraxial focusing of azimuthal-variant vector beams. Opt. Express 2012, 20, 17684-17694. [CrossRef] [PubMed]

88. Gu, B.; Xu, D.; Pan, Y.; Cui, Y. Nonparaxial propagation and focusing properties of azimuthal-variant vector fields diffracted by an annular aperture. J. Opt. Soc. Am. A 2014, 31, 1657-1665. [CrossRef] [PubMed]

89. Deng, D.; Guo, Q.; Wu, L.; Yang, X. Propagation of radially polarized elegant light beams. J. Opt. Soc. Am. B 2007, 24, 636-643. [CrossRef]

90. Deng, D.; Guo, Q. Analytical vectorial structure of radially polarized light beams. Opt. Lett. 2007, 32, 2711-2713. [CrossRef] [PubMed]

91. Banerjee, P.P.; Cook, G.; Evans, D.R. A q-parameter approach to analysis of propagation, focusing, and waveguiding of radially polarized Gaussian beams. J. Opt. Soc. Am. A 2009, 26, 1366-1374. [CrossRef]

92. Borghi, R.; Santarsiero, M. Nonparaxial propagation of spirally polarized optical beams. J. Opt. Soc. Am. A 2004, 21, 2029-2037. [CrossRef]

93. Cui, X.; Wang, C.; Jia, X. Nonparaxial propagation of vector vortex beams diffracted by a circular aperture. J. Opt. Soc. Am. A 2019, 36, 115-123. [CrossRef]

94. Guo, L.; Chen, L.; Lin, R.; Zhang, M.; Dong, Y.; Chen, Y.; Cai, Y. Nonparaxial Propagation Properties of Specially Correlated Radially Polarized Beams in Free Space. Appl. Sci. 2019, 9, 997. [CrossRef]

95. Petrov, N.I. Nonparaxial focusing of wave beams in a graded-index medium. Rus. J. Quantum Electr. 1999, 29, 249-255. [CrossRef]

96. Petrov, N.I. Focusing of beams into subwavelength area in an inhomogeneous medium. Opt. Express 2001, 9, 658-673. [CrossRef] [PubMed]

97. Petrov, N.I. Macroscopic quantum effects for classical light. Phys. Rev. A 2014, 90, 043814. [CrossRef]

98. Petrov, N.I. Reflection and transmission of strongly focused light beams at a dielectric interface. J. Mod. Opt. 2005, 52, 1545-1556. [CrossRef]

99. Norrman, A.; Gil, J.J.; Friberg, A.T.; Setälä, T. Polarimetric nonregularity of evanescent waves. Opt. Lett. 2019, 44, 215-218. [CrossRef]

100. Petrov, N.I. Evanescent and propagating fields of a strongly focused beam. J. Opt. Soc. Am. A 2003, 20, 2385-2389. [CrossRef]

101. Voipio, T.; Setala, T.; Friberg, A.T. Partial polarization theory of pulsed optical beams. J. Opt. Soc. Am. A 2013, 30, 71-81. [CrossRef]

102. Petrov, N.I.; Danilov, V.A.; Popov, V.V.; Usievich, B.A. Large positive and negative Goos-Hänchen shifts near the surface plasmon resonance in subwavelength grating. Opt. Express 2020, 28, 7552-7564. [CrossRef]

103. Petrov, N.I. Splitting the bandwidth of a frustrated total internal reflection filter with nanoparticle inclusions. OSA Contin. 2020, 3 , 2591-2601. [CrossRef]

104. Petrov, N.I. Frustrated-total-internal-reflection-based thin-film color separator. Opt. Lett. 2007, 32, 2744-2746. [CrossRef]

105. Petrov, N.I. Achromatic Polarization rotator. Appl. Opt. 2007, 46, 6340-6343. [CrossRef] [PubMed]

106. Petrov, N.I.; Nikitin, V.G.; Danilov, V.A.; Popov, V.V.; Usievich, B.A. Subwavelength diffractive color beam combiner. Appl. Opt. 2014, 53, 5740-5744. [CrossRef] [PubMed]

107. Petrov, N.; Khromov, M.; Sokolov, Y. Multi-View 3D Integral Imaging Systems Using Projectors and Mobile Devices. Photonics 2021, 8, 331. [CrossRef]

108. Petrov, N.I.; Petrova, G.N. Diffraction of partially-coherent light beams by microlens arrays. Opt. Express 2017, 25, 22545-22564. [CrossRef]

109. Soifer, V.A.; Kharitonov, S.I.; Khonina, S.N.; Strelkov, Y.S.; Porfirev, A.P. Spiral Caustics of Vortex Beams. Photonics $2021,8,24$. [CrossRef]

110. Kazanskiy, N.L.; Khonina, S.N.; Karpeev, S.V.; Porfirev, A.P. Diffractive optical elements for multiplexing structured laser beams. Quantum Electron. 2020, 50, 629-635. [CrossRef]

111. Bezus, E.A.; Bykov, D.A.; Doskolovich, L.L. Integrated diffraction gratings on the Bloch surface wave platform supporting bound states in the continuum. Nanophotonics 2021, 10, 4331-4340. [CrossRef]

112. Dorrah, A.H.; Tamagnone, M.; Rubin, N.A.; Zaidi, A.; Capasso, F. Introducing Berry phase gradients along the optical path via propagation-dependent polarization transformations. Nanophotonics 2021, 11, 19. [CrossRef]

113. Petrov, N.I. Evolution of Berry's phase in a graded-index medium. Phys. Lett. A 1997, 234, 239-250. [CrossRef]

114. Bliokh, K.Y.; Nori, F. Transverse and longitudinal angular momenta of light. Phys. Rep. 2015, 592, 1-38. [CrossRef]

115. Eismann, J.S.; Nicholls, L.H.; Roth, D.J.; Alonso, M.A.; Banzer, P.; Rodríguez-Fortuño, F.J.; Zayats, A.V.; Nori, F.; Bliokh, K.Y. Transverse spinning of unpolarized light. Nat. Photonics 2021, 15, 156-161. [CrossRef]

116. Ketara, M.L.; Kobayashi, H.; Brasselet, E. Sensitive vectorial optomechanical footprint of light in soft condensed matter. Nat. Photonics 2021, 15, 121-124. [CrossRef]

117. Cardano, F.; Marrucci, L. Longitudinal fields and transverse rotations. Nat. Photonics 2021, 15, 72-74. [CrossRef] 
118. Lindfors, K.; Priimagi, A.; Setälä, T.; Shevchenko, A.; Friberg, A.T.; Kaivola, M. Local polarization of tightly focused unpolarized light. Nat. Photonics 2007, 1, 228-231. [CrossRef]

119. Marrucci, L. Spin gives direction. Nat. Photonics 2014, 11, 9-10. [CrossRef]

120. Wang, J.; Sciarrino, F.; Laing, A.; Thomson, M.G. Integrated photonic quantum technologies. Nat. Photonics 2020, 14, $273-284$. [CrossRef] 